\title{
Identity change and informed consent
}

\section{OPEN ACCESS}

\author{
Correspondence to \\ Dr Karsten Witt, Institut \\ für Philosophie, Universität \\ Duisburg-Essen, Universitätsstr. \\ 12, Essen 45141, Germany; \\ karsten.witt@uni-due.de
}

Received 11 May 2016

Revised 10 January 2017

Accepted 25 January 2017

Published Online First

17 March 2017

\section{Karsten Witt}

quality of life he repeatedly expresses satisfaction with his current situation. ${ }^{\mathrm{i}}$

Many bioethicists immersed in the ethics of DBS describe cases like that of Mr Smith as involving a 'change of (personal) identity'. One might wonder what is conveyed in such statements. Since logic tells us that there cannot be changes of numerical identity, coherent talk of 'identity changes' must be about 'identity' in a different sense (cf. ref. 6, p. 356). Most bioethicists working on DBS and similarly effective techniques accept this line of thinking. Accordingly, they distinguish between 'numerical identity' and 'identity' in a different sense and point out that they are concerned with the latter. Although there is disagreement over the details, 'identity' in this second sense is commonly taken to denote a collection or set of characteristics that make a person distinctive and the elements of which would figure in an appropriate answer to the question 'Who am I?' (cf. ref. 7 pp. 77-83). I take it that this set comprises a person's central projects, plans, beliefs, commitments, values and behavioural traits (cf. ref. 8, pp. 41, 47). In what follows, I will use '(personal) identity' and its cognates exclusively in the 'Who am I' sense of the term.

Identity changes in this sense are not only discussed in the literature on DBS. Within the medical context they are also claimed to occur in connection with brain-tissue transplantation, facial allograft transplantation, organ transplantation, brain-computer interfaces, brain chips, neurosurgery and psychopharmacology. ${ }^{\text {ii }}$ I use the catch-all term 'identity-changing interventions' for them, 'identity-changing' meaning that the intervention may, with probability $\mathrm{p}, 0<\mathrm{p} \leq 1$, cause a change in the patient's identity.

In this paper, I will not be concerned with the magnitude of p. Nor will I question the empirical claim that the interventions just listed really $d o$ bring about changes in identity in certain patients. I assume that the majority of commentators are correct in believing that they do. What interests me is what follows from this, ethically speaking. How can medical interventions like DBS, psychopharmacology, organ transplantation, and so on, be justified given the truth of the widespread assumption that they can sometimes change the patient's identity?

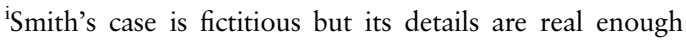
see, for example, the cases described in refs 1: p. 486, 2 pp. 7-8, 3: p. 19, 4: p. 466, and 5.

${ }^{1 i}$ See refs. 9-15, respectively. Identity changes also occur outside the medical context and might thus play a role in accounts of morality more generally. It is unclear to me how the position I am going to develop here relates to these broader issues, partly because of significant unclarities about the nature and role of consent outside of biomedical ethics; cf. ref. 16: section 9 .
}

To cite: Witt K. J Med Ethics 2017;43:384-390. with his wife. This, however, does not bother Smith. Quite the contrary: when asked about his 
In modern bioethics, it is generally agreed that the ethical justification of medical interventions presupposes the patient's informed consent prior to the intervention. ${ }^{\text {iii }}$ This agreement is rooted in the shared conviction that an informed consent is an autonomous authorisation and that medical practice should protect or respect the patient's fundamental right to autonomous choice (cf. ref. 17 , p. $206 ; 18$, p. 121). In this paper, I accept these widely held claims.

But what does informed consent to identity-changing interventions amount to? Are the conditions that have to be met in order for an approval to count as an instance of informed consent the same as in non-identity-changing interventions? Convenient as an affirmative answer would be, the truth, I think, is more complex. Informed consent to identity-changing interventions is importantly different from informed consent to normal interventions. Or so I will argue.

The paper is organised as follows: In the section 'The standard conception', I briefly sketch the conception of informed consent to identity-changing interventions most frequently found in the literature today. In the section 'A problem for the standard conception', I formulate an objection that purports to show that conception to be seriously lacking, a claim which I will further discuss in the section 'Defending the standard conception'. In the section 'Refining the standard conception: the perspective-sensitive approach', I propose a refinement of the standard conception, the 'perspective-sensitive account of informed consent', and explain its various elements. Finally, in the section 'Conclusion', I take stock and briefly list directions for further research.

\section{THE STANDARD CONCEPTION}

The standard conception of informed consent to identitychanging interventions rests squarely on the idea of comprehensive information. More precisely, it holds that, prior to the intervention, the patient is to be informed not only about the effects that typically go along with invasive medical interventions (intraoperational risks, curative effects, effects on symptoms control, enhancement effects, risks of rejection of transplanted tissue, etc), but also about the possibility of identity change.

Two variants of the standard conception might be distinguished, a sparse and a more generous one. Most adherents of the standard conception support the sparse variant. They agree with Nir Lipsman and colleagues, who write that it is

imperative to disclose the potential for personality change ... in order to complete the informed consent process. (Ref. 14, p. 381$)^{\text {iv }}$

Observers that are more generous might be willing to follow Debra Mathew's proposal that the patient has to be informed about all effects 'related to personal identity'. (Ref. 1, p. 487) Among other things, this would require them to be informed about marital conflicts that may result from identity changes. (Refs. 1, p. 490; 2, p. 10)

This is correct as far as it goes. But does it go far enough? I don't think so. Identity changes and their consequences must play a more sophisticated role in a patient's deliberations in order for her consent to an identity-changing intervention to

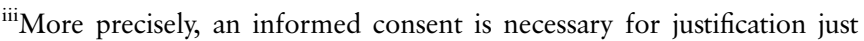
in case patients are (i) competent and (ii) willing to exercise their right to informed consent. In this paper, I assume that (i) and (ii) hold. That is, I won't deal with the incompetent or the so-called 'problem of waivers'.

${ }^{\text {iv }}$ See refs. 10, p. 452,19 , p. 5,20 , p. 580 , and 21, p. 411.
}

count as an instance of informed consent. Treating identity changes and their consequences like 'normal' side effects invariably leads to trouble.

\section{A PROBLEM FOR THE STANDARD CONCEPTION}

To see what I have in mind, consider a simple question: what happens when a person's identity changes? As explained above, I follow conventional wisdom in taking a person's identity to be a collection of characteristics that comprises the person's central projects, plans, beliefs, commitments, values and basic behavioural traits. An identity change involves a change in one or more of these central mental elements. But a person whose identity is altered changes not only with regard to certain core beliefs, attitudes or personality traits. Arguably, she also changes with regard to how she evaluates or judges things.

Consider the peculiar case of Mr Smith. Through DBS, he changes from a modest, diligent person and loving husband to an extraverted egotist who has no interest in the projects that were of central importance for him before the intervention. In light of these changes, we can expect him not only to behave but also to judge things differently before the intervention and afterwards. Adopting a somewhat imprecise but well-established terminology in ethics, the judgments delivered from his preintervention perspective (his 'preintervention judgments') can be expected to differ from those delivered from his postintervention perspective (his 'postintervention judgments'). ${ }^{\mathrm{v}}$ We thus expect him, from his preintervention perspective, to welcome the prospect of being less of a burden for his wife, but to be deeply worried about upcoming familial and professional problems, and to reject the prospect of becoming an outgoing, egocentric person. And we expect him, from his postintervention perspective, to be happy with the latter while being indifferent to the former consequences of the intervention. But then we have judgments that seem to be relevant for the justification of the intervention that pull in opposite directions. Call this the problem of 'opposing judgments'.

Adherents of the standard conception have generally overlooked the possibility of opposing judgments in identitychanging interventions. This is problematic. Arguably, consenting to an identity-changing intervention without paying due heed to the possibility of opposing judgments is not an instance of informed consent. Neither is a refusal an informed refusal. ${ }^{\text {vi }}$ This becomes clear when we consider two kinds of mistake, one blatant and one subtle, that patients can commit if they do not include opposing judgments and, more generally, the judgmental consequences of identity changes into their deliberations about identity-changing interventions.

First, the blatant mistake. If a patient thinks about undergoing an identity-changing intervention exclusively from the preintervention perspective, she might agree to what she later, from her postintervention perspective, rejects. Quite obviously, such a decision can have tragic consequences, especially in irreversible

'Since I will frequently talk about 'perspectives' in what follows, a caveat is in order: We all know that our outlook may change not only due to changes in identity, but also due to other factors like good music, meditation, empathy, stress, love, anger, and so on. What changes in these and other cases is not what I call 'a person's perspective'. Rather, a person's perspective, as I use the term, is her way of understanding and evaluating things that is based on her identity at that time or phase of life. For a similar usage, see, for example, refs. 22, pp. 236-237 and 8, p. 47.

${ }^{\mathrm{v}}$ For the close links between informed consent and informed refusal, see refs. 17, 18, pp. 124, 136. In what follows, I will largely keep my focus on informed consent. 
interventions. The person might consent to effects she would rather not experience when they actually occur. To put it more dramatically, she might unknowingly, and with the best of intentions, condemn herself to an enduring loss in quality of life or even lifelong suffering.

Next, the subtle mistake. The patient might, from her preintervention perspective, reject what she would have approved of had she undergone the identity-changing intervention. A decision like this might not have such tragic consequences as the other. Still, it can lead to opportunities remaining unexplored and a life that is worse than it could have been. This last point might be doubted. Think again of Mr Smith and assume that he expects the intervention to improve his motor abilities but render him egocentric and extraverted. Now, imagine that in light of this information and some thorough reasoning based on his preintervention identity Smith decides to reject the intervention. It might be asked how this could ever be problematic given that he correctly anticipates that the intervention will make him egocentric and extraverted and that he doesn't want to be like that. But the rhetorical nature of this question is misguided. Smith's decision is based solely on his preintervention perception of the anticipated changes. It leaves out his postintervention satisfaction. Because of this he misses the opportunity of becoming someone enjoying these changes and, more generally, the possibility of leading an overall better life (cf. ref. 23, pp. 76-77).

It is important not to confuse the blatant and the subtle mistake with another kind of mistake that can have similar effects but has nothing specifically to do with identity-changing interventions. Mistakes of this kind arise in cases in which patients overlook 'ordinary' bits of information about the consequences of an intervention such that their consent or refusal may not count as informed. To keep to my earlier example, a patient deliberating about whether to undergo a certain intervention might unknowingly condemn herself to lifelong suffering not only by overlooking a change in identity, but simply by overlooking 'ordinary' side effects such as chronic backache or hypertension. Since the badness of these effects is not dependent on a change in judgmental perspective, I will ignore them in what follows.

So much for the deleterious consequences of blatant and the subtle mistake and their specific link to identity-changing interventions. I have already claimed that overlooking these mistakes prevents an informed choice of the patient. Now I am in a position to spell out my argument for this claim. It consists of two premises: first, an informed choice requires the patient to understand and appraise all relevant information about the intervention. Second, it is not the case that a patient who overlooks the possibility of either the blatant or the subtle mistake has understood and appraised all relevant information about the intervention. Thus, patients deciding about an identity-changing intervention without considering the possibility of either the blatant or the subtle mistake cannot make an informed choice. Since the standard conception doesn't provide the conceptual tools to accommodate the two mistakes, it is unsatisfactory and in need of refinement.

\section{DEFENDING THE STANDARD CONCEPTION}

Have I been too quick? While the first premise of the above argument is part of the bioethical mainstream that I accepted at the outset, the second premise might be more controversial. Do patients really have to consider the possibility of opposing judgments in general and the blatant and the subtle mistake in particular for their consent to be informed? Can't they legitimise an identity-changing intervention without thinking about these things? A defender of the standard conception will try to convince us that they can. Here are two ways in which she might proceed.

First, she might claim that in the case of identity-changing interventions patients lack the information necessary to put themselves in their later shoes (so to speak). Therefore, they cannot avoid the blatant and the subtle mistake. And therefore, it is wrong to blame the standard conception for not providing the conceptual tools to do so.

However, it seems to me that our current situation is not so deplorable, epistemically speaking. For one, case studies on identity-changing interventions suggest that patients change only in a limited number of ways. Even today, experienced physicians and their staff can make fairly reliable guesses about an intervention's identity effects on patients with a given personality type and inform their patients about this. Moreover, in the near future, physicians will probably have at their disposal effective predictor profiles allowing for more systematic estimates of this sort (cf. ref. 24). Finally, we should remind ourselves that a considerable fraction of identity-changing interventions are reversible. At least in principle one could gather accurate information about an intervention's identity effects in a given case by deliberately reversing or stopping the intervention for a certain time. There might be moral and pragmatic problems with this proposal in certain cases and I lack the space to discuss them here. But my hunch is that arrangements for planned reversals might be ethically sound when the patient consents to them before the intervention (from her preintervention perspective) and when her consent is informed in the sense that it is based on case studies about identity change or the estimates of an experienced physician. Be this as it may, I think we have sufficient grounds to doubt whether patients and their physicians today are in the epistemically deprived situation that is crucial for the present objection to work. Besides, we can reasonably expect access to better information in the near future such that looking for a timely alternative to the standard conception surely is the sensible thing to do.

Second, the defender of the standard approach might try to argue that even if information about identity changes is available to patients, they do not have to take it into account for their consent to count as 'informed'. We must distinguish between informed consent and what might be called 'ideally rational' consent. ${ }^{\text {vii }}$ While the latter may well require the consideration of identity changes and the mistakes they might lead to, the former doesn't. Therefore, even if it fails as a conception of ideally rational consent, the standard conception is an appropriate account of informed consent.

One way in which this thought might be substantiated is to argue that many patients will find it hard to anticipate postintervention life. Therefore, if such anticipation were required, only a small fraction of patients could give their informed consent to identity-changing interventions. But an adequate conception of informed consent cannot have such excluding consequences. Therefore, we should favour the standard conception.

I am unsure whether an adequate conception of informed consent must never have excluding consequences for a majority of patients, but I will accept that assumption in what follows. I would prefer to cast doubt on the claim that many patients cannot appropriately anticipate postintervention life. Why should they be so limited? People frequently empathise with others and seem quite good at it. At least it would be surprising

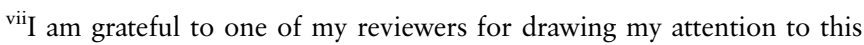
distinction. 
if it turned out that most of these attempts fail. Especially with regard to people close to them they can successfully put themselves into someone else's shoes. Much prudential advice is based on that ability. And usually such advice is helpful. Apparently, then, many people are able to take up another person's perspective. Why not in their own case? Moreover, as pointed out above, in many identity-changing interventions patients change only in a limited number of ways such that their physicians can inform them accordingly. Moreover, typical changes are well documented on video, in case studies and in books. Going through some of this material will help patients to imagine postintervention life. If planned reversals of identitychanging interventions for the purpose of gathering information are permissible, this will further facilitate their job. The claim that most patients are unable to figure out postintervention life is untenable against this background.

Another way of defending the standard conception turns on the special conditions that must be in place for the blatant and the subtle mistake to occur. For the blatant mistake to occur, life after the intervention has to be worse than before; for the subtle mistake, the patient has to believe that life after the intervention will be worse than before. Assuming that patients thinking about undergoing identity-changing interventions suffer to a significant degree, and assuming that identity changes usually go along with significant improvements of the symptoms causing the suffering, either condition will only very rarely be met. But for consent to count as informed, the patient need only understand the 'central facts' of the decision she is facing (cf. ref. 18, p. 132). So why make so much fuss over the two mistakes? Shouldn't we rather hold on to the standard conception and ignore these theoretical subtleties?

However, I doubt the alleged rareness of the mistakes. For one, my discussion is not necessarily confined to identitychanging therapies in a narrow sense. It might easily be extended to include identity-changing enhancement interventions. At least for this subclass of identity-changing interventions, the above assumptions that patients thinking about undergoing identity-changing interventions suffer to a significant degree and that identity-changing interventions typically alleviate this suffering are misplaced. Furthermore, I have until now embedded my discussion of the two mistakes in a fairly specific decisional context in which there is only one medical intervention on offer such that the patient has the choice between undergoing it and leaving everything as it is. Putting enhancement interventions to one side, it is probably true that in this specific context the two mistakes only rarely occur. When suffering due to disease and suffering due to identity change are the only outcomes on offer, situations in which the latter will (be believed to) exceed the former will indeed be rare. But this 'binary' context is, as I said, special. In many situations in which identity-changing interventions are at stake, other treatments are also available such that the patient faces alternatives to doing nothing (the default option) and embracing the identity-changing intervention. In these wider contexts, analogues of the blatant and the subtle mistake will probably be more frequent. As to the blatant mistake: choosing the identity-changing intervention might be no worse than the default option, yet worse than some medium-range alternative. As to the subtle mistake: patients might not see the identitychanging intervention as worse than the default option yet, again, worse than an alternative. Thus, there is reason to doubt the present objection even for the subclass of identitychanging interventions that count as therapies in the narrow sense of the term.
As far as I can see, then, none of the objections succeeds. The standard conception cannot be saved from the difficulties ensuing from opposing judgments and the mistakes they might lead to. Since the only way to avoid these difficulties is to systematically include the patient's postintervention judgment in the informed consent procedure, it is high time to look for refinement of the standard conception.

\section{REFINING THE STANDARD CONCEPTION: THE PERSPECTIVE-SENSITIVE APPROACH}

If the standard conception is to avoid the unhappy consequences of opposing judgments, it must be significantly broadened and refined. My proposal for meeting this challenge is what I call the 'perspective-sensitive approach' to informed consent. It consists of three necessary conditions, which I propose should complement existing conceptions of informed consent, for example, the elaborate conception defended by Tom Beauchamp and James Childress (cf. ref. 18, pp. 121-141).

I start with the argument for the first two conditions of the perspective-sensitive approach. In the section 'A problem for the standard conception', I argued that there are problems in identity-changing interventions that cannot satisfactorily be dealt with by the (unrefined) standard conception. The reason for this shortcoming is that it does not systematically factor in the different perspectives that go along with identity-changing interventions. Consider again the blatant and the subtle mistake. Both occur because postintervention effects are judged from the preintervention perspective. It is this grouping together of preintervention perspective and (expected ${ }^{\text {viii }}$ ) postintervention effects that results in an overvaluation or undervaluation of postintervention quality of life, leading to the blatant and the subtle mistake respectively. The only way to avoid both mistakes is to let the patients include their preintervention and postintervention perspectives when deciding whether to have an identity-changing intervention. But how exactly should this be done?

As concerns quality of life, the answer is obvious. The patient should figure out her preintervention quality of life from her preintervention perspective and her postintervention quality of life from her postintervention perspective. Ensuring this match of perspectives and well-being is a reliable way to curtail the blatant and the subtle mistake and is therefore one element of the account I will develop here.

However, more needs to be done. We have not yet considered the evaluation of the identity change itself. Opposing judgments are not necessarily restricted to quality of life. They can (and often will) include diverging judgments about identity changes. How should these be dealt with? Consider again our introductory case. Smith rejects any identity changes from his preintervention perspective while endorsing the change that actually happens to him from the postintervention perspective. Has he changed for the better or for the worse? Which of his two judgments is authoritative for answering this question? In other words, what is the right perspective for evaluating his identity change? Or should both perspectives have an equal say?

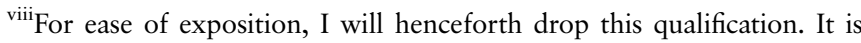
expressly not my aim to blur the distinction between decisions about identity-changing interventions under certainty and under uncertainty. I am well aware that the perspective-sensitive account must also function under conditions of uncertainty if it is to be widely applicable in clinical practice. Still, to keep matters manageable I will not elaborate these points here. Sometimes the way from thorough philosophical thinking to clinical application is too long for a single paper.
} 
Here is an argument for the claim that it is the preintervention perspective from which patients should assess identity changes: first, approval is informed consent just in case it is autonomous consent. Second, in case of opposing judgments about an identity change, only an assessment from the preintervention perspective can be part of autonomous consent. Therefore, in case of opposing judgments about an identity change, only an assessment from the preintervention perspective can be part of informed consent.

I won't discuss the argument's first premise here because it is part and parcel of the bioethical mainstream that I have accepted in the section 'Introduction'. What about the second premise? Is it true that identity changes can only be autonomously assessed from the preintervention perspective? When we look at Joseph Smith again, this is how it seems to be: Smith's postintervention approval of the identity change appears to be caused by the same mechanism that caused the change itself. The stimulation not only altered his personality and his central projects, but also made him approve of those changes. If this is what happens, there is a relevant sense in which the approval is not his own: like the change itself, it is not under his control. Instead of being the result of rational, independent deliberation on his side, it has been brought about by the intervention. Therefore, it counts as heteronomous and to that extent unsuitable for informed consent. Consequently, the identity change should be assessed from Smith's preintervention perspective alone.

This line of thought is based on what has been called a 'historical' account of the nature of autonomy. According to the historical account, a necessary condition for a mental attitude to be autonomous (or, as some say, authentic) is for it to have the right kind of history. ${ }^{\text {ix }}$ What is necessary and sufficient for a history to be of the 'right kind' is controversial among friends of the historical account. However, there appears to be broad agreement that it must be free of non-rational influences that are so far-reaching as to determine how the person will judge them on reflection (cf. refs. 28 , p. $16 ; 29$, p. 14). In the case of Smith's postintervention approval, this condition appears to be violated. And it is plausible to assume that it may be violated in other postintervention assessments of identity-changing interventions too. Therefore, informed consent to such interventions seems to require that identity changes be assessed from the preintervention and not the postintervention perspective.

The argument presupposes that the preintervention identity is always an appropriate vantage point for the assessment of identity changes. However, this assumption is too strong: ${ }^{\mathrm{x}}$ some patients' preintervention identities might be 'disease-shaped' such that they qualify as heteronomous according to the historical account outlined above (cf. ref. 30, p. 491). Or their illness and the stigma accompanying it might render them 'overly deferential' to the wishes, opinions or 'discriminatory narratives' of

\footnotetext{
${ }^{\mathrm{ix}} \mathrm{A}$ historical account of autonomy is not as popular among contemporary bioethicists as it is outside of bioethics. But it has its defenders; cf. refs. 25 , p. 375,18 , pp. $113-114,26$, p. 565 . Since it leaves room for non-historical necessary conditions, it is compatible with the idea that incompetence or the experience of alienation from one's current identity (or relevant parts thereof) thwart or reduce autonomy. It can thus deal with the currently much discussed 'dilemma' between authenticity and autonomy in identity-changing interventions in an elegant and illuminating way. For the supposed dilemma and a promising way to avoid it, see refs. 27 and 26, respectively.

${ }^{\mathrm{x}} \mathrm{I}$ am grateful to another anonymous reviewer for urging me to elaborate on this point.
}

the people around them (cf. refs. 31, p. 523; 26, p. 566). In these and other cases, we might reasonably doubt the autonomy of judgments from the preintervention perspective. At any rate, they will probably be less autonomous than judgments from the postintervention perspective if the intervention is medically successful.

Although I do not doubt the reality of such cases, I will not deal with them in what follows. Is this a problem? That depends on whether they force us to conclude that an adequate conception of informed consent to identity-changing interventions must allow for the possibility that identity changes be evaluated solely from the postintervention perspective (or, for that matter, from both the preintervention and postintervention perspectives). Perhaps this is what these cases teach us. Then what I am going to say here is not about informed consent to identitychanging interventions as such, but only about consent in all and only those cases in which the patient is able to render the relevant autonomous judgments from the preintervention perspective. But perhaps these cases do not imply anything like this. Perhaps patients whose identities are disease-shaped or who are overly deferential to others are not autonomous enough to give their informed consent in the first place, and the justification of identity-changing interventions in their case must rely on other moral resources. If this is correct, the conception of informed consent I am going to develop will be as comprehensive as one could wish.

I will not try to decide the matter here. It might therefore turn out that the scope of my account is limited: to those patients whose preintervention judgments about the identity change (and, as I will later argue, about the relative weight of identity and quality-of-life considerations) are at least as autonomous as their postintervention judgments about these matters. Given this caveat, I now introduce the first two conditions of the perspective-sensitive approach:

A patient's approval of an identity-changing intervention is an informed consent only if it is based on

(C1) an assessment of her preintervention quality of life and the identity change from her preintervention perspective, and

(C2) an assessment of her (expected) postintervention quality of life from her postintervention perspective. ${ }^{\mathrm{xi}}$

$\mathrm{C} 1$ and $\mathrm{C} 2$ require the patient to assess certain things from the preintervention and other things from the postintervention perspective. This makes good sense because, as the idea of informed consent is usually and plausibly understood, the patient's consent should rest upon an all-things-considered judgment: apart from the usual practical difficulties, this task is straightforward in normal interventions in which the patient's perspective remains unaffected. But how should the patient, even in principle, arrive at an all-things-considered judgment when this assumption no longer holds and two perspectives are involved? Call this the 'consolidation question'. Answering it is the last step on my way to the perspective-sensitive approach.

To arrive at an answer to the consolidation question, we need a way to somehow integrate the patient's perspectival judgments made according to $\mathrm{C} 1$ and $\mathrm{C} 2$. How should this be done? Frederic Schick, in discussing the systematically related decision-theoretic problem of endogenous changes, makes a promising proposal:

${ }^{\mathrm{xi}}$ For an account of informed consent to identity-changing interventions featuring variants of $\mathrm{C} 1$ and $\mathrm{C} 2$, see ref. 32, pp. 14-15. 
Let $x$ and $y$ be the only options you have. ... [Y]ou could choose $x$ only if your $x$-consequent self ranked the prospect of $x$ no lower than your $y$-consequent self ranked the prospect of $y$. (Ref. ${ }^{23}$, pp. $76-77$

Freely adapting Schick's schema to our needs, it becomes: you can consent to an identity-changing intervention only if the quality your life has after the intervention, according to the postintervention perspective, is no lower than the quality your life has before (or without) the intervention according to the preintervention perspective. Here is one way of motivating this stimulating thought. Deleting the references to the preintervention and postintervention perspective, we see that the adapted schema is really just a variant of the well-known advice that the patient should choose the option with the highest expected quality of life. Surely, it is as reasonable to aim for a good life in medical decisions that might involve an identity change as it is in decisions that do not.

The adapted schema gives a partial answer to the consolidation question: it advises us not to prioritise between the quality-of-life judgments. In a way, this is felicitous because it simplifies the analysis. Prioritising would seem to require a prioritising judgment by the patient. Since this judgment would have to be rendered from one perspective or other, I would have to say from which perspective and why. Alternatively, prioritising might depend in some objective way on the patient's identity, as Parfit and McMahan might be taken to argue. ${ }^{\text {xii }}$ This, too, would raise intricate problems. As against this, the adapted schema doesn't require and, in fact, doesn't allow for prioritisation. Rather, it asks for a non-perspectival balancing of preintervention and postintervention quality of life, giving equal weight to the quality-of-life judgments rendered from the two perspectives involved.

However, the adapted schema leaves a problematic gap. It contains no advice about how to include identity changes in the overall picture. How might this be done? In an attempt to provide an answer to this question, I make two assumptions. First, I assume that, much as a patient's quality of life can improve or deteriorate, so can her identity (as judged from her preintervention perspective). Second, I assume that, at least in principle, improvements or deteriorations of identity can be weighed against improvements or deteriorations in quality of life. The latter assumption in particular may not be wholly uncontroversial but, as far as I can judge, it is widely accepted in the literature (cf. refs. 35, p. 290; 2, p. 8). At any rate I won't defend it here.

With the adapted schema and the two assumptions in place, my former question about the inclusion of judgments about identity changes in the patient's deliberative process becomes a question about how changes in identity and changes in quality of life should be weighed against each other. Call this the 'special consolidation question'. The special consolidation question is of particular interest when the judgments on identity change and change in quality of life point in opposite directions, as illustrated in table 1 .

Looking at this table, one might, for instance, ask how the patient should decide when her identity deteriorates while her quality of life improves. The right answer seems to depend on

\footnotetext{
xii According to roughly Parfitian lines, instead of treating postintervention quality of life on a par with preintervention quality of life, the former should be discounted to the extent that the patient's postintervention identity differs from her preintervention identity; cf. refs. 33, pp. 313-314; 34, pp. 79-82.
}

\begin{tabular}{|c|c|c|}
\hline & $\begin{array}{l}\text { Postintervention identity as } \\
\text { compared with preintervention } \\
\text { identity }\end{array}$ & $\begin{array}{l}\text { Balance of postintervention and } \\
\text { preintervention quality of life }\end{array}$ \\
\hline 1 & Deterioration & Improvement \\
\hline 2 & Improvement & Deterioration \\
\hline
\end{tabular}

the individual patient's weighing preference. If she cares a lot about her identity, the items in the left-hand column will count for more than those in the right-hand column. She will, for instance, be deeply troubled by the prospect of being changed in a way she doesn't approve of, where gains in quality of life may not tip the scales in favour of the identity-changing intervention. In contrast to this, if a patient doesn't care much about who she is or becomes, the entries in the left-hand column will impress her to a far lesser degree than those in the right-hand column.

However, there is a problem with the preference-based answer to the special consolidation question. It cannot be ruled out that the degree to which the patient cares for her identity relative to her quality of life will be affected by the intervention. Thus, a patient whose preintervention life is characterised by extensive 'landscape-gardening of the self' (ref. 36, p. 132 might be turned into someone who is more interested in her quality of life and vice versa. So the patient may have no single weighing preference that can inform an answer to the special consolidation question, but one such preference before the intervention and another afterwards. Is there a principled way of deciding between the two?

I think there is. The preference pattern that should inform the special consolidation question is the one the patient herself favours when looking at the issue from her preintervention perspective. If she thinks that the change in the relative weights of identity and quality of life is a change for the better, she should rely on her postintervention preference; if she thinks the change is for the worse, she should stick to her preintervention preference. This proposal corresponds to my treatment of identity changes. In both cases, patients should assess interventioninduced changes from their preintervention perspective (again, given the caveat above). This is no accident because there is an important link between the two. We have seen that it is incompatible with autonomy to assess identity changes from the patient's postintervention perspective because this might give rise to the intervention's establishing its own validation. But if the evaluation of the identity change must not be established by the intervention, the same must go for the weight it is assigned in the decision-making process. The reason is that otherwise the evaluation of the identity change can be rendered completely ineffective as a result of a weighing preference that strongly favours quality of life over identity and that has, in a relevant sense, been established by the intervention itself. Thus, if it is wrong to assess identity changes from the postintervention perspective, the same must go for assessments of changes in weighing preference.

These considerations conclude my exploration of the perspective-sensitive account. Summing up my previous results, here is the account in full bloom:

(Perspective-sensitive account) A patient's approval of an identitychanging intervention is an informed consent only if the patient's consent is based on

(C1) an assessment of her preintervention quality of life and the identity change from her preintervention perspective, and 
(C2) an assessment of her postintervention quality of life from her postintervention perspective, provided that

(C3) preintervention and postintervention quality of life are weighted equally, and the weight assigned to the identity change in the deliberative process is that which the patient endorses when considering her weighing preferences from her preintervention perspective.

As I have argued above, this approach is superior to the approach standardly favoured in the literature.

\section{CONCLUSION}

On the foregoing pages I have focused on a kind of intervention that is at the same time fascinating and disturbing: identitychanging interventions. My guiding question was how such interventions can be ethically justified within the bounds of contemporary bioethical mainstream that places great weight on the patient's informed consent. The answer most frequently given in the relevant literature today is that patients should be informed about the identity effects, thus treating changes in identity as simply a further effect of the intervention. I have argued that this approach is seriously lacking because it misses important complexities in identity changes and is consequently bound to run into mistakes. As a remedy I have proposed the perspective-sensitive account, which provides the conceptual resources to systematically deliberate about and give valid consent to identity-changing interventions.

In spite of my in-depth treatment of these matters, several important issues have not been addressed. I have only begun to pursue the possibility that the patient's preintervention perspective might sometimes be less autonomous than her postintervention perspective; I have mentioned but not discussed Parfitian reservations against the weighing scheme that is part of the perspective-sensitive account (cf. n. 12); and I have omitted certain difficulties for the implementation of the perspectivesensitive account in clinical practice (cf. n. 8). A comprehensive account of informed consent to identity-changing interventions will have to consider these further points.

Acknowledgements I thank Catharine Lewis for helping me devise my introductory case, Oliver Hallich, Achim Lohmar, and Lars Dänzer for valuable comments on different versions of the paper and the German Research Foundation for funding the project "Grounding and persistence. Personal identity in bioethics". An earlier version of the article has been awarded the DGPPN Prize for Ethics and Philosophy in Psychiatry and Psychotherapy 2016. (The DGPPN is the German Association for Psychiatry, Psychotherapy and Psychosomatics).

Funding Deutsche Forschungsgemeinschaft (WI 4519/2-1).

Competing interests None declared.

Provenance and peer review Not commissioned; externally peer reviewed.

Open Access This is an Open Access article distributed in accordance with the Creative Commons Attribution Non Commercial (CC BY-NC 4.0) license, which permits others to distribute, remix, adapt, build upon this work non-commercially, and license their derivative works on different terms, provided the original work is properly cited and the use is non-commercial. See: http://creativecommons.org/ licenses/by-nc/4.0/

\section{REFERENCES}

1 Mathews DJH. Deep brain stimulation, personal identity and policy. Int Rev Psychiatry 2011;23:486-92.

2 Müller S, Christen M. Deep brain stimulation in Parkinsonian patients-ethical evaluation of cognitive, affective, and behavioral sequelae. AJOB Neurosci 2011:2:3-13.
3 Mackenzie R. Who should hold the remote for the new me? Cognitive, affective, and behavioral side effects of DBS and authentic choices over future personalities. AJOB Neurosci 2011;2:18-20.

4 Lipsman N, Glannon W. Brain, mind and machine: what are the implications of deep brain stimulation for perceptions of personal identity, agency and free will? Bioethics 2013;27:465-70.

5 Lewis CJ, Maier F, Horstkötter N, et al. Subjectively perceived personality and mood changes associated with subthalamic stimulation in patients with Parkinson's disease. Psychol Med 2015;45:73-85.

6 Olson ET. Personal identity. In: Stich SP, ed. The Blackwell guide to philosophy of mind. Malden, MA: Blackwell, 2003:352-68.

7 DeGrazia D. Human identity and bioethics. Cambridge: Cambridge University Press, 2005.

8 Shoemaker S. Identity and identities. Daedalus 2006;135:40-8.

9 Northoff G. Do brain tissue transplants alter personal identity? Inadequacies of some "standard" arguments. J Med Ethics 1996;22:174-80.

10 Swindell JS. Facial allograft transplantation, personal identity and subjectivity. J Med Ethics 2007;33:449-53.

11 Svenaeus F. Organ transplantation and personal identity: how does loss and change of organs affect the self? J Med Philos 2012;37:139-58.

12 Kotchetkov IS, Hwang BY, Appelboom G, et al. Brain-computer interfaces: military, neurosurgical, and ethical perspective. Neurosurgical Focus 2010;28:E25.

13 Maguire GQ, McGee EM. Implantable brain chips? Time for debate. Hastings Center Report 1999:29:7-13.

14 Lipsman N, Zener R, Bernstein M. Personal identity, enhancement and neurosurgery: a qualitative study in applied neuroethics. Bioethics 2009;23: 375-83.

15 Kramer PD. Listening to Prozac. New York: Penguin Books, 1997.

16 Eyal N. Informed consent. In Zalta EN. ed, The Stanford Encyclopedia of Philosophy. 2012. http://plato.stanford.edu/entries/informed-consent/\#ConOthDom

17 Ridley DT. Informed consent, informed refusal, informed choice-what is it that makes a patient's medical treatment decisions informed? Med Law 2001:20:205-14

18 Beauchamp TL, Childress JF. Principles of biomedical ethics. New York: Oxford University Press, 2013

19 Focquaert $F$, DeRidder $D$. Direct intervention in the brain: ethical issues concerning personal identity. J Ethics Ment Health 2009:4:1-7.

20 Bell E, Mathieu G, Racine E. Preparing the ethical future of deep brain stimulation. Surg Neurol 2009;72:577-86, discussion 586.

21 Gilbert F. The burden of normality: from 'chronically ill' to 'symptom free'. New ethical challenges for deep brain stimulation postoperative treatment. J Med Ethics 2012:38:408-12.

22 Williams BAO. The idea of equality. In: Williams BAO, ed. Problems of the self. Philosophical papers 1956-1972. Cambridge: Cambridge University Press, 1999:230-49.

23 Schick F. Making choices. A recasting of decision theory. Cambridge: Cambridge University Press, 1997.

24 Smeding HMM, Speelman JD, Huizenga HM, et al. Predictors of cognitive and psychosocial outcome after STN DBS in Parkinson's Disease. J Neurol Neurosurg Psychiatry 2011;82:754-60.

25 Quante M. Precedent autonomy and personal identity. Kennedy Inst Ethics J 1999:9:365-81.

26 Wardrope A. Authenticity and autonomy in deep-brain stimulation. J Med Ethics 2014; 40:563-6

27 Kraemer F. Authenticity or autonomy? When deep brain stimulation causes a dilemma. J Med Ethics 2013;39:757-60.

28 Taylor JS. Introduction. In: Taylor JS, ed. Personal autonomy. New essays on personal autonomy and its role in contemporary moral philosophy. Cambridge: Cambridge University Press, 2005:1-29.

29 Christman J. Autonomy, history, and the subject of justice. Soc Theory Pract 2007:33:1-26.

30 Kraemer F. Me, myself and my brain implant: deep brain stimulation raises questions of personal authenticity and alienation. Neuroethics 2013;6:483-97.

31 Baylis F. 'I am who I am': On the perceived threats to personal identity from deep brain stimulation. Neuroethics 2013;6:513-26.

32 Witt K. Das Identitätsproblem der tiefen Hirnstimulation und einige seiner praktischen Implikationen. Ethik in der Medizin 2013;25:5-18.

33 Parfit D. Reasons and persons. Oxford: Clarendon Press, 1984.

34 McMahan J. The ethics of killing. Problems at the margins of life. New York: Oxford University Press, 2002.

35 Glannon W. Stimulating brains, altering minds. J Med Ethics 2009;35:289-92.

36 Glover J. l: The philosophy and psychology of personal identity. London: Penguin Books, 1988. 